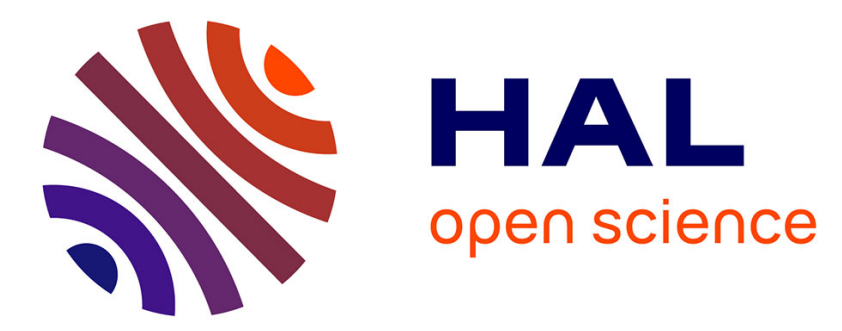

\title{
Chasing unphysical TD-DFT excited states in transition metal complexes with a simple diagnostic tool
}

\author{
Federica Maschietto, Marco Campetella, Juan Sanz García, Carlo Adamo, \\ Ilaria Ciofini
}

\section{- To cite this version:}

Federica Maschietto, Marco Campetella, Juan Sanz García, Carlo Adamo, Ilaria Ciofini. Chasing unphysical TD-DFT excited states in transition metal complexes with a simple diagnostic tool. Journal of Chemical Physics, 2021, 154 (20), pp.204102. 10.1063/5.0050680 . hal-03297245

\section{HAL Id: hal-03297245 \\ https://hal.science/hal-03297245}

Submitted on 23 Jul 2021

HAL is a multi-disciplinary open access archive for the deposit and dissemination of scientific research documents, whether they are published or not. The documents may come from teaching and research institutions in France or abroad, or from public or private research centers.
L'archive ouverte pluridisciplinaire HAL, est destinée au dépôt et à la diffusion de documents scientifiques de niveau recherche, publiés ou non, émanant des établissements d'enseignement et de recherche français ou étrangers, des laboratoires publics ou privés. 


\section{Chasing unphysical TD-DFT excited states in transition}

\section{metal complexes with a simple diagnostic tool}

Federica Maschietto $^{1,2}$, Marco Campetella ${ }^{1,3}$, Juan Sanz-García ${ }^{1,4}$, Carlo Adamo ${ }^{1,5}$, and Ilaria Ciofini $^{1 *}$

Chimie ParisTech, PSL University, CNRS, Institute of Chemistry for Life and Health Sciences, Theoretical Chemistry and Modelling, 75005 Paris, France; Department of Chemistry, Yale University, New Haven, Connecticut 06520-8107, United States; Consiglio Nazionale Delle Ricerche (CNR) SPIN, area di ricerca di Tor vergata, via del fosso del cavaliere 100, 00133 Roma; MSME, Univ. Gustave Eiffel, CNRS UMR 8208, F-77454 Marne-la-Vallée, France; Institut Universitaire de France 103 Bd Saint-Michel, F-75005 Paris - France

\section{Corresponding Authors}

*E-mail: ilaria.ciofini@chimieparistech.psl.eu, federica.maschietto@yale.edu

ORCID Numbers:

Federica Maschietto: 0000-0002-5995-2765

Marco Campetella: 0000-0002-1853-6685

Juan Sanz-García: 0000-0002-3175-0061

Carlo Adamo: 0000-0002-2638-2735

Ilaria Ciofini: 0000-0002-5391-4522 


\section{ABSTRACT}

Transition Metal Complexes (TMC) are known for the rich variety of their excited states showing different nature and degree of locality. Describing the energies of these excited states with the same degree of accuracy is still problematic when using Time Dependent Density Functional Theory in conjunction with the most current Density Functional Approximations. In particular, the presence of unphysically low lying excited states possessing a relevant Charge Transfer (CT) character may significantly affect the spectra computed at such level of theory and, more relevantly, the interpretation of their photophysical behavior.

In this work we propose an improved version the $M_{\mathrm{AC}}$ index, recently proposed by the authors and collaborators, as a simple and computationally inexpensive diagnostic tool that can be used for the detection and correction of the unphysically predicted low lying excited states. The analysis, performed on five prototype TMC, shows that spurious and ghost states can appear in a wide spectral range and that it is difficult to detect them only on the basis of their CT extent. Indeed, both delocalization of the excited state and charge transfer extent are criteria that must be combined, as in the $M_{\mathrm{AC}}$ index, to detect unphysical states. 


\section{Introduction}

The prediction of the nature and properties of excited states of transition metal complexes (TMCs) is a fertile research area. ${ }^{1-9}$ Due to their peculiar photochemical and photophysical properties, ${ }^{10-12}$ organometallic complexes of several metals are nowadays exploited in many different fields, spanning from photovoltaics ${ }^{13-17}$ to medical and biological applications. ${ }^{18-23}$

However, photophysical and photochemical processes occurring in TMC are intrinsically complex and extremely interesting to study, yet difficult to understand based on sole experimental investigations. Indeed, the use of theoretical approaches is crucial to correctly model the behavior of such complexes upon irradiation and to acquire a realistic description of their excited state manifold. ${ }^{1-9,16-18}$ TMCs, though, cumulate most of the complexities inherent to electronic structure investigations such as a high density of low lying electronic states of a different character (from charge transfer -CT- to locally excited -LE-), the presence of nearly degenerate states, and/or of relevant relativistic effects. A proper description of these compounds is thus a challenging task for any quantum method.

On one hand, post-Hartree-Fock methods, such as complete-active-space self consistent field (CASSCF), ${ }^{24}$ restricted-active-space SCF (RASSCF) variants ${ }^{25}$ often improved by adding a perturbative correction (CASPT2), ${ }^{26}$ have proven to yield high accuracy in describing both vertical absorption and photochemical behavior of metal complexes, ${ }^{27}$ although somehow limited in their domain of applicability by their computational burden. Moreover, these methods require a selection of a relevant and system-dependent active space, which makes them impractical and of heavy usage for routine applications.

On the other hand, Density functional theory (DFT) and its Time-Dependent extension (TD-DFT) benefit from their favorable scaling and cost to accuracy ratio which has determined their widespread diffusion for the description of metal-based complexes. ${ }^{1}$ Besides, density rooted approaches limit the user dependency to the choice of exchange-correlation functional to be used, which in practice renders these methods easy to use, though impressively accurate in the description of structural and spectroscopic properties of such class of compounds, at least for what concerns the electronic ground state and the lowest excited states. Not surprisingly, TD-DFT calculations and the corresponding simulated spectra are increasingly used to support and interpret experimental findings, providing useful insights on the nature of the observed transitions and thus providing a precious help to interpret the photophysics of such compounds. , $^{5-8,16-17,22-23}$ 
In most cases, the reliability of the theoretical method is assessed based on the agreement between the computed and the experimentally observed spectra. However, this experimental/theoretical spectra matching may result from an accidental compensation of errors or hide some methodological issues. ${ }^{28,29}$ In this respect, it is well known that TD-DFT may fail to describe excitations with a significant through-space charge-transfer (CT) character depending on the exchange-correlation functional used. ${ }^{28,29}$ Indeed, this failure could be related to the selfinteraction error ${ }^{32,33}$, the incorrect asymptotic behavior, ${ }^{34,35}$ and the missing derivative discontinuity $^{36}$ of the exchange-correlation (xc) functional used. These errors particularly affect functionals resting on the generalized gradient approximation (GGA) ${ }^{37}$ while being partially cured by the introduction of Hartree-Fock exchange, including global $(\mathrm{GH})^{31}$ and range separated (RSH) hybrids, double hybrids, ${ }^{38,39}$ or purposely tuned hybrids. ${ }^{40}$

These known limitations of density functional approximations ${ }^{30-31,41-42}$ (DFAs) may lead de-facto to an erroneous description of CT excited states and to the prediction of unphysically low lying excited states. This applies with no exception not only to organic push-pull dyes on intramolecular CT excitations, as already largely documented in the literature, ${ }^{29}$ but also to metal-containing complexes. ${ }^{1}$ In the latter case, the errors are even more relevant for the interpretation of their photophysical properties since both CT and LE states very close in energy may be present but unequally affected by the limitations of the DFA used.

To diagnostic these problems a number of descriptors have been developed over the years, with the double role of identifying the nature of the excited electronic states and, possibly, to evaluate the TD-DFT error, which could be intrinsically related to it. An overview of these indexes is outside the scope of the present paper, here we only recall that they have been successfully and widely applied in literature and that they are nowadays available in several codes. ${ }^{43-51}$

A less explored field concerns the validation of such indexes for TMCs. Indeed, in this class of compounds a rich variety of excited states showing a different degree of locality exists, including for instance metal-centered (MC), ligand-centered (LC), metal-to-ligand-charge transfer (MLCT), ligand-to-metal-charge-transfer (LMCT), intra-ligand-charge-transfer (ILCT), and ligand-toligand-charge-transfer (LLCT) states. Most often, these excited states are identified by inspection of the associated natural transition orbitals ${ }^{52}$ or by the use of CT descriptors ${ }^{53-57}$ always assuming that their relative energy is correctly predicted. Nonetheless, this latter point is of crucial importance when aiming to accurately interpret the photophysics and photochemistry of TMC. 
In order to (partially) fulfill this gap, in the present contribution we examine the lowest excitedstate levels of five octahedrally- substituted $\mathrm{Ru}(\mathrm{II})$ complexes, shown in Figure 1.

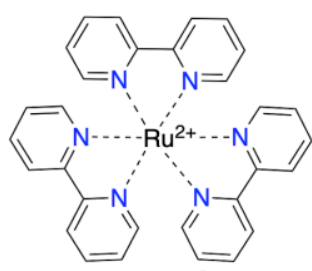

$\left[\mathrm{Ru}(\mathrm{bpy})_{3}\right]^{2+}(\mathbf{1})$

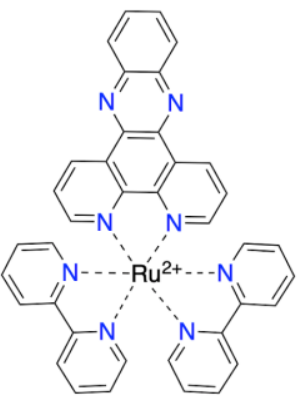

$\left[\mathrm{Ru}(\mathrm{bpy})_{2}(\mathrm{dppz})\right]^{2+}(\mathbf{3})$

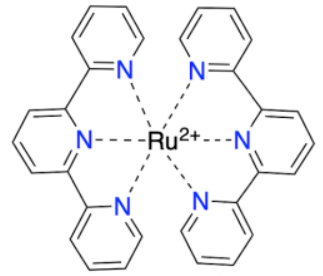

$\left[\mathrm{Ru}(\text { tpy })_{2}\right]^{2+}(2)$

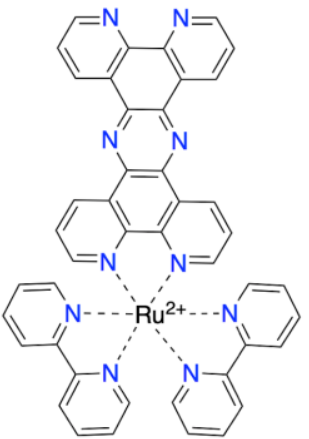

$\left[\mathrm{Ru}(\mathrm{bpy})_{2}(\mathrm{tpphz})\right]^{2+}(\mathbf{4})$
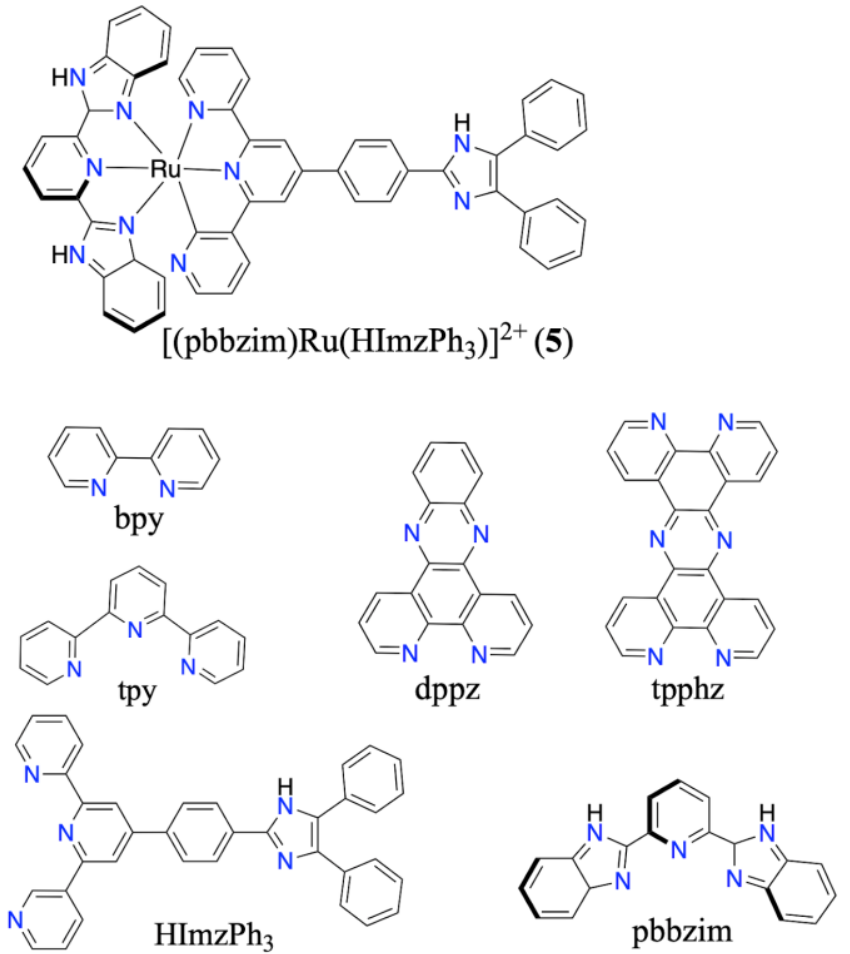

Figure 1: Structures of the $\mathrm{Ru}(\mathrm{II})$ complexes investigated in this study together with the representation of the bidentate and tridentate ligands involved.

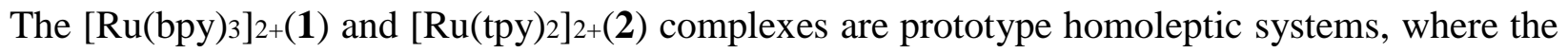
$\mathrm{Ru}$ atom is coordinated either with three bpy (2,2'-bipyridine), or two tpy (2,2:2',6'-terpyridine) ligands. The photophysics of these systems has extensively been studied in literature ${ }^{58,59}$ and they constitute the skeleton of many of the Ru complexes developed for various applications. ${ }^{20,60,61}$ The three heteroleptic $\left([\mathrm{Ru}(\mathrm{bpy}) 2(\mathrm{dppz})]_{2+(3)}, \quad[\mathrm{Ru}(\mathrm{bpy}) 2(\mathrm{tpphz})]_{2+(4)}\right.$ and $\quad[($ pbbzim $) \operatorname{Ru}($ tpyHImzPh3) $]_{2+(5)}$ are derivatives of the two homoleptic scaffolds, substituted with the dppz (dipyrido[3,2- a:2',3'-c]phenazine), tpphz (tetrapyrido[3,2-a:2',3'-c:3",2"'-h:2"',3"'-j]phenazine), pbbzim (2,6-bis(benzimidazole-2-yl)pyridine) and tpy-HImzPh3 (4'-[4-(4,5-diphenyl-1Himidazol-2- yl)-phenyl]-[2,2':6',2"] terpyridine) ligands, respectively expected to promote the formation of low lying excited states of MLCT character. ${ }^{59,62-64}$ 
All these compounds have been previously experimentally characterized ${ }^{58,59,62-64}$ and they will be used to assess the quality of a diagnostic tool enabling to evaluate the quality of the predicted electronic transitions using three largely applied DFAs.

The purpose of this study is indeed to propose a diagnostic index enabling to spot when TD-DFT fails in the description of excited states, and, more generally, to provide a simple strategy to assess on the fly the validity of different DFAs, in the context of the spectral properties prediction of TMCs. This index should enable to disclose the presence of unphysical states and to derive a possible correction of the wrongly predicted transition energies. The present analysis, beside its theoretical interest in evaluating xc functionals performances, may be worthwhile to be considered while designing and optimizing metal complexes based on the outcomes of TD-DFT predictions, especially when targeting charge-transfer or charge-separated excited states for experimental applications.

\section{Analysis of vertical excitations using the $M_{\mathrm{AC}}$ index}

As mentioned above, a direct consequence of the use of approximated xc functionals in the framework of TD-DFT is that excitations with substantial through-space CT character may be poorly described with the consequence that their excitation energies can be severely underestimated. These excited states are the so-called "ghost" states. Mostly they correspond to through-space electronic transitions with significant CT character and negligible hole-particle overlap, which appear at very low energies. ${ }^{30,31}$ The presence of such spurious states, while not necessarily affecting the spectral shape, can affect the interpretation and prediction of the photophysical behavior of molecular systems implying, for instance, that an energetically higher bright state could decay non-radiatively into the lower CT states, leading to an electron-transfer quenching of the excited state fluorescence. In this respect, the limitations of a selected DFA have an impact that is much larger than its numerical performances (i.e. the error in the computed transition energies w.r.t. a given reference), leading to a wrong interpretation of the photophysical behavior of the system under investigation. These ghost states generally have vanishing oscillator strength. Although energy underestimation and negligible oscillator strength can be used as criteria to detect such unphysical states, these indicators may be not sufficient to unambiguously identify them as "ghosts". Furthermore, as the CT error strongly depends on the chosen DFA, the degree by which these are mistaken varies significantly: a given DFA for a given system may provide a 
correct description and energy estimation of excited states with limited CT character while incorrectly underestimate the energy of other, longer range, CT states.

To address the difficulty in identifying and eventually correct excited states of different nature in the TD-DFT framework, we have recently introduced a diagnostic index, the so-called $M_{\mathrm{AC}} .{ }^{65}$ The $M_{\mathrm{AC}}$ index is based on the formula originally proposed by Mulliken ${ }^{66}$ to estimate a lower bound to the energy associated with an intermolecular CT state $\left(\omega_{\mathrm{CT}}\right)$ in the case of Donor to Acceptor dyad and lately discussed in the framework of TD-DFT by Head-Gordon and collaborators ${ }^{30}$

$$
\omega_{C T}=I P_{D}-E A_{A}-\frac{1}{R}
$$

Here, $\mathrm{R}$ represents the distance between the electron donor/acceptor moieties, $I P_{\mathrm{D}}$ is the ionization potential of the donor, $E A \mathrm{~A}$ is the electron affinity of the acceptor. $1 / R$ is thus the electrostatic interaction between the hole and the electron (in atomic units) after a donor-to-acceptor CT excitation.

In the original $M_{\mathrm{AC}}$ index, ${ }^{65}$ these quantities were computed from Kohn-Sham (KS) orbital eigenvalues $\left(I P_{\mathrm{D}}\right.$ and $\left.E A_{\mathrm{D}}\right)$ and the hole-electron distance $(\mathrm{R})$ was estimated by the $D_{\mathrm{CT}}$ index. ${ }^{45}$ This index provides a measure of the hole-electron distance from the barycenters of the charge densities corresponding to an increase and decrease of the electron density upon electronic excitation. A more extensive description of this index can be found in Supporting Information. As such, it represents the effective (average) charge/hole distance produced in an electronic excitation and therefore can be used, as in the original $M_{\mathrm{AC}}$ formulation, to provide a realistic estimate of the electrostatic contribution to the electronic transition energy (last term of eq. 1) both in the case of intra and intermolecular electron transfer.

In order to apply the $M_{\mathrm{AC}}$ index to TMCs and aiming at ameliorating the estimate of the first two terms of Eq. 1 with respect to our previous formulation ${ }^{65}$ the following expression will be used here:

$$
M_{A C}=\frac{\sum_{i a} c_{i a}^{2}\left(\varepsilon_{a}^{D F A-H F}-\varepsilon_{i}^{D F A-H F}\right)}{\sum_{i a} c_{i a}^{2}}-\frac{1}{R}
$$

Indeed, to improve the original $M_{\mathrm{AC}}$, for each computed excited state $I P_{\mathrm{D}}$ and $E A \mathrm{~A}$ are estimated by a weighted average of the eigenvalues of the contributing KS molecular orbitals being computed at the Hartree-Fock level, on top of the converged DFT orbitals. This allows one to correct the underestimation of TD-DFT-virtual orbitals eigenvalues. Indeed, by this single HF cycle, we mitigate the known KS-bias, which compromises the energy of the empty orbitals with 
excessively negative correlation energy values (in an absolute sense), artificially lowering their values. The improved hole and particle orbital energies relative to the transition considered are denoted here as $\varepsilon_{i}^{D F A-H F}$ and, $\varepsilon_{a}^{D F A-H F}$ respectively. The weights, $c_{i a}$, are the CI coefficients obtained as solutions of TD-DFT equations. ${ }^{67}$ The procedure is easy and fast, as it requires merely to perform an estimation at HF level of the molecular orbital energies on top of the converged Kohn-Sham orbitals.

As in the case of the previous formulation, ${ }^{65}$ by construction the $M_{\mathrm{AC}}$ index depends on the quality of ground and excited state densities used to compute the $D_{\mathrm{CT}}$. Concerning the excited states electronic density, recent publications have highlighted the importance of density relaxation effects in TD-DFT, ${ }^{68,69}$ which can be estimated by employing the so-called Z-vector formalism. ${ }^{70}$ With this type of computation one performs a post-linear response treatment of the excited state calculation, resulting in the addition of an off-diagonal contribution to the transition-density which accounts for the redistribution of the electron density in the orbital space. The resulting quantity is the so-called "relaxed" difference density matrix, which unlike the "unrelaxed" density matrix accounts, at least partially, for the orbital relaxation following the hole/particle generation. The projection in real space of the relaxed and unrelaxed difference density matrices defines what we usually refer to as relaxed and unrelaxed densities. ${ }^{28,71}$ Both density definitions can be used to evaluate the $D_{\mathrm{CT}}$ and yield the corresponding indexes ${ }^{\mathrm{R}} D_{\mathrm{CT}}$ and ${ }^{\mathrm{U}} D_{\mathrm{CT}}$. The former reflects the spatial extent associated to a given transition, where the electronic density is allowed to gradually change and adapt to the final configuration, while the latter reproduces the CT distance, measured directly upon vertical excitation. In other words, the relaxed ( $\left.{ }^{\mathrm{R}} D_{\mathrm{CT}}\right)$, unlike its unrelaxed ( $\left.{ }^{\mathrm{U}} D_{\mathrm{CT}}\right)$ counterpart accounts for the redistribution of the electronic charge due to the excitation. Clearly, this double definition of the $D_{\mathrm{CT}}$ leads to two distinct $M_{\mathrm{AC}}$ indexes: the ${ }^{\mathrm{U}} M_{\mathrm{AC}}$ and ${ }^{\mathrm{R}} M_{\mathrm{AC}}$. The effect of the use of these two variants is detailed below.

The $M_{\mathrm{AC}}$ index, as defined in Eq. 2, defines a lower bound to the excitation energy associated with a given charge transfer transition and can be used to diagnostic the presence of unphysically predicted low-lying excited states due to artifacts of the xc functional applied. A given TD-DFT transition will be, therefore, identified as a ghost if its energy is lower than the corresponding $M_{\mathrm{AC}}$ index, while proper CT excitations will have an energy greater than the $M_{\mathrm{AC}}$ value. Thus, for each electronic transition two cases can be distinguished: 


$$
\begin{aligned}
& \mathrm{E}_{\mathrm{TD}-\mathrm{DFT}}<M_{\mathrm{AC}} \rightarrow \text { unphysical CT state } \\
& \mathrm{E}_{\mathrm{TD}-\mathrm{DFT}}>M_{\mathrm{AC}} \rightarrow \text { real CT state. }
\end{aligned}
$$

Clearly it follows from Eq. 1 -and from its equivalent formulation in Eq. 2- that the condition in eq. 4 is always fulfilled in the case of transitions of local character that are possessing a negligible charge-transfer character and for which the $\mathrm{R}$ (or the $D_{\mathrm{CT}}$ ) value is close to zero. Indeed, the Mulliken formula is relevant only for transitions of CT type and in the following we will focus on such kinds of excitations.

In the case of states predicted at unphysical low energy, that is states that fulfill the criteria of eq. 3 , an additional distinction is made here: those with zero oscillator strength will be classified as "ghost" states and labeled as "G". By contrast, CT excitations that have a non-vanishing oscillator strength, but still appear too low in energy, will be referred to as "spurious" states and labeled as "S".

\section{Computational details}

All electronic structure calculations were performed using the Gaussian 16 quantum package. ${ }^{72}$ Starting from the crystallographic X-ray structures, complexes 1 to 5 were optimized in acetonitrile using the Polarizable Continuum Model (PCM) ${ }^{73}$ following the same protocol as described in Ref. 74. We employed DFT using the standard hybrid functional B3LYP ${ }^{75,76}$ with the 6-31G(d,p) basis set with one set of $d$ polarization functions for the second-row elements and a set of p polarization functions for the hydrogen atoms. ${ }^{77}$ For the ruthenium atom we used the LANL08 effective core potential (including 28 core electrons) and associated basis set for valence electrons. ${ }^{78}$ Additionally, we performed vibrational frequency calculations - at the same level of theory - to ensure that all structures correspond to minima. Although the assumption of considering in all cases B3LYP structures can induce bias and errors when aiming at comparing with experimental data, in the present case it has the advantage of allowing to directly compare the behavior of the different functionals in predicting vertical excited states energies and nature, which is indeed the main goal of the present paper.

The UV-Vis absorption spectra of all complexes were then computed using the TD-DFT approach and three different functionals, namely the hybrid functional B3LYP, ${ }^{75,76}$ the long-range corrected 
CAM-B3LYP ${ }^{78}$ functional and the hybrid functional PBE0,${ }^{80}$ using the previously defined basis set. In TDDFT calculations only spin-allowed (singlet-singlet) transitions were considered. Excited states were also computed using the Configuration Interaction with Singles excitation (CIS) approach, since as it includes $100 \%$ HF exchange, it follows that no unphysical states should be found. As shown by the tables reported in SI, indeed no ghost states are detected by the $M_{\mathrm{AC}}$ analysis at the CIS level thus providing a proof of the reliability and consistency of this index. Nonetheless due to the incomplete treatment of correlation effects, CIS is normally overestimating transition energies and the corresponding transition energy values are here only considered as a ghost-free reference more than reference to assess the accuracy of TD-DFT excitation energy values and will thus not be discussed in detail in the text.

The natural transition orbitals (NTO) relative to the thirty lowest transitions were also computed to inspect the character of the transitions. These last are collected in Figures SI.3 to SI.7. Finally, to investigate the presence of methodological artifacts, related to the choice of the exchangecorrelation functional, we computed the MAC index on the lowest thirty transitions of each complex, both using unrelaxed and relaxed electron densities. Accordingly, the $\mathrm{U}$ and $\mathrm{R}$ labels, in the following, will denote that the corresponding MAC value is obtained using either of the two density definitions. This analysis was performed using an in-house developed tool.

For complexes 3, 4 and 5 difference density plots, computed using relaxed densities for each transition are collected in Figures SI.8 to SI.10.

\section{Results and discussion}

In this work we will show how the diagnostic index defined as in eq. 2 can provide relevant insights to detect ghost and spurious CT states in TMCs focusing on two global hybrid (B3LYP and PBE0) and one range-separated (CAM-B3LYP) functionals, all widely employed in recent literature for TMC, and analyzing the lowest lying excited states of the five selected metal complexes depicted in Figure 1.

Figure 2 shows the computed and experimental absorption profiles of the five selected $\mathrm{Ru}$ (II) compounds in acetonitrile. When $\pi$-accepting ligands, such as polypyridyl, are coordinated to $\mathrm{Ru}(\mathrm{II})$, the complex exhibits intense singlet-singlet MLCT transitions in the visible region. ${ }^{60}$ This behavior is typical for both tris-bidentate and bis-tridentate complexes, although a slight red-shift of the maximum absorption wavelength is often observed for the more rod like bis-tridentate 
ones. ${ }^{18}$ The intense charge transfer MLCT transitions are also more easily formed in the presence of ligands able to delocalize density at the excited state far from the metal center, that is to give rise to excited states with larger CT distance. Thus, in Figure 2, the complexes bearing ligands with higher electron-accepting capabilities show more intense and red-shifted MLCT bands. Clearly metal-centered and ligand-centered transitions can also be identified in the electronic spectrum of each of the compounds. A list of all computed transitions and associated assignments is provided in Supporting Information in Table SI.1 to SI.5. together with a representation of all NTOs involved reported in Figure SI.3 to SI.7.

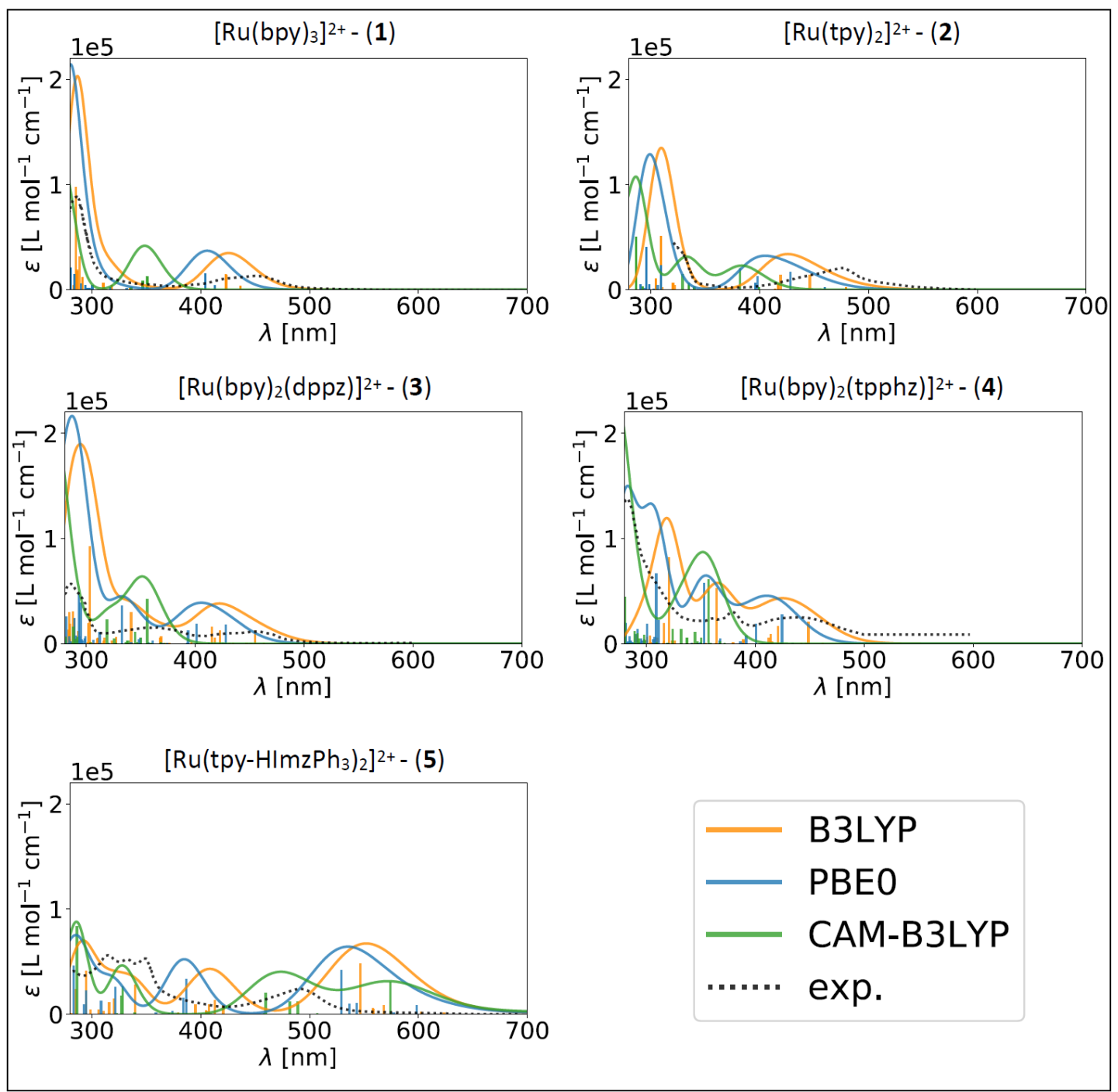

Figure 2: Absorption spectrum of $\mathbf{1}, \mathbf{2}, \mathbf{3}, \mathbf{4}$, and $\mathbf{5}$ computed using different functionals, along with the corresponding experimental spectra, retrieved from references. ${ }^{47,49-52}$ The simulated spectra are computed by a Gaussian convolution of the computed vertical transitions using a full-width half maximum value of $0.4 \mathrm{eV}$. Computational details are provided in Supporting Information. 
The absorption profiles of $\mathbf{1}$, calculated with different methods, is shown in the upper left panel of Figure 2. In agreement with previous works, ${ }^{12,58}$ the broad band around 400-500 nm corresponds to multiple MLCT transitions $-\mathrm{d}^{6}(\mathrm{Ru})$ to $\pi *(\mathrm{bpy})$. Indeed, in this region of the spectrum, the most intense transitions are ascribed to the population of the degenerate excited states ES7 and ES8 both in the case of B3LYP (at $424 \mathrm{~nm}$ ) and PBE0 (at $405 \mathrm{~nm}$ ), these states being of MLCT character. The same transitions appear higher in energy - at $352 \mathrm{~nm}$ and correspond to excited states ES5 and ES6 - in the case of CAM-B3LYP, in agreement with previous reports showing a overestimation of transition energies at range separated level in the case of $\mathrm{Ru}$ polypyridine complexes. ${ }^{81}$ The natural transition orbitals (NTOs) reported in Supporting Information, Figures SI.3 and the corresponding computed energy values and oscillator strengths, reported in Table SI.1, support these assignments. Of note, and as expected due to the presence of unsubstituted bpy ligands, although being of MLCT character these transitions are rather local, the charge transfer extent being quite limited as evident from the associated $D_{\mathrm{CT}}$ values.

A qualitatively similar description can be made for complex 2, the first intense transition, independently of the method considered, being predicted to be related to the population of excited state ES5, an MLCT state characterized by a relatively low CT distance and computed at $447 \mathrm{~nm}$, $427 \mathrm{~nm}$ and $382 \mathrm{~nm}$ at B3LYP, PBE0 and CAM-B3LYP levels, respectively (see Figure SI.2). In the case of GHs only, a second transition of comparable intensity also contributes to the first absorption band at $420 \mathrm{~nm}$ and $398 \mathrm{~nm}$, for B3LYP and PBE0, respectively.

In agreement with the experimental data, overall the spectral features of complex $\mathbf{3}$ are not significantly different from those of complex $\mathbf{1}$, the associated bands being calculated slightly redshifted both using GH functionals and CAM-B3LYP. In this case for both GHs three electronic transitions mostly contribute to the first absorption band. These transitions involve the population of excited states ES4, ES9 and ES11 in the case of B3LYP (computed at $455 \mathrm{~nm}, 423 \mathrm{~nm}$ and 416 nm, respectively) and excited states ES4, ES9 and ES11 (computed at 429 nm, 402 nm and 393 $\mathrm{nm}$, respectively) at PBE0 level. In the case of CAM-B3LYP on the other hand a very intense transition at $355 \mathrm{~nm}$ determines the absorption band maxima while a shoulder at higher energies appears due to other less intense transitions. All these transitions correspond to MLCT bands although not localized on the same moieties of the molecule (see for instance Figure SI.5). 
In the case of complex 4, the situation is similar: several transitions of different MLCT type (see Figure SI.5) but of comparable intensities are computed to contribute to the first absorption band at $445 \mathrm{~nm}, 421 \mathrm{~nm}$ and $414 \mathrm{~nm}$ at B3LYP level and $417 \mathrm{~nm}, 401 \mathrm{~nm}$ and $353 \mathrm{~nm}$ at PBE0 level (see Table SI.4). On the other hand, using CAM-B3LYP a single dominant transition at $357 \mathrm{~nm}$ gives rise to the first absorption band. This leads to redshifted absorption bands in the case of GHs with respect to the $\mathrm{RSH}$.

The calculated absorption profile of $\mathbf{5}$ is qualitatively different from the one of complexes $\mathbf{1 - 4}$, independently of the DFA considered. The first intense band calculated with both GHs is due to the convolution of several bright transitions of different MLCT character, the most intense being in both cases related to be population of excited state ES11 (computed at $548 \mathrm{~nm}$ and $530 \mathrm{~nm}$ at B3LYP and PBE0, respectively, see Table SI.5). Contrary to the 1-4, the first intense transition is computed at lower energy at CAM-B3LYP level, at $576 \mathrm{~nm}$, though showing the same M to tpy MLCT character.

In order to evaluate if the computed spectra may be influenced by the presence of spurious states and if the interpretation of the photophysical properties of these compounds may be misleading due to the prediction of low-lying ghost states, a diagnostics analysis based on the $M_{\mathrm{AC}}$ index was performed. Its results are graphically reported in Figures 3 and 4 and listed in Tables SI.1 to SI.5 together with the previously described transition energies, oscillator strength and character. Both ${ }^{\mathrm{U}} M_{\mathrm{AC}}$ and ${ }^{\mathrm{R}} M_{\mathrm{AC}}$ were calculated at different levels of theory. The use of unrelaxed densities, rather than the costlier relaxed ones, is indeed particularly convenient in the case of metal complexes, where the number of excited states to analyze is often large. Unrelaxed densities can be computed all at once, in a single TD-DFT calculation, for all desired vertical states at a given geometry, with significant savings in terms of time and resources. Relaxation effects, by contrast, need to be computed separately for each excited state, thus being more computationally expensive but with the advantage of delivering a refined description of the density distribution upon transition. As shown in our previous work ${ }^{68}$ where we investigated the impact of the relaxation on CT states for different DFAs, the two approaches are equivalent only when aiming for a qualitative interpretation of the nature of electronic excitations. Indeed, if both relaxed and unrelaxed densities can in principle be used to compute the $M_{\mathrm{AC}}$ index, the latter -unrelaxed densities- will, in general, result in an overestimation of the $\mathrm{CT}$ character and consequent exaggeration on the number of ghost and spurious states. Therefore, an inexpensive diagnostic analysis based on unrelaxed 
densities can be interesting as a preliminary screening, but shall be confirmed by computing relaxation effects for those states that are classified as unphysical in the first place.

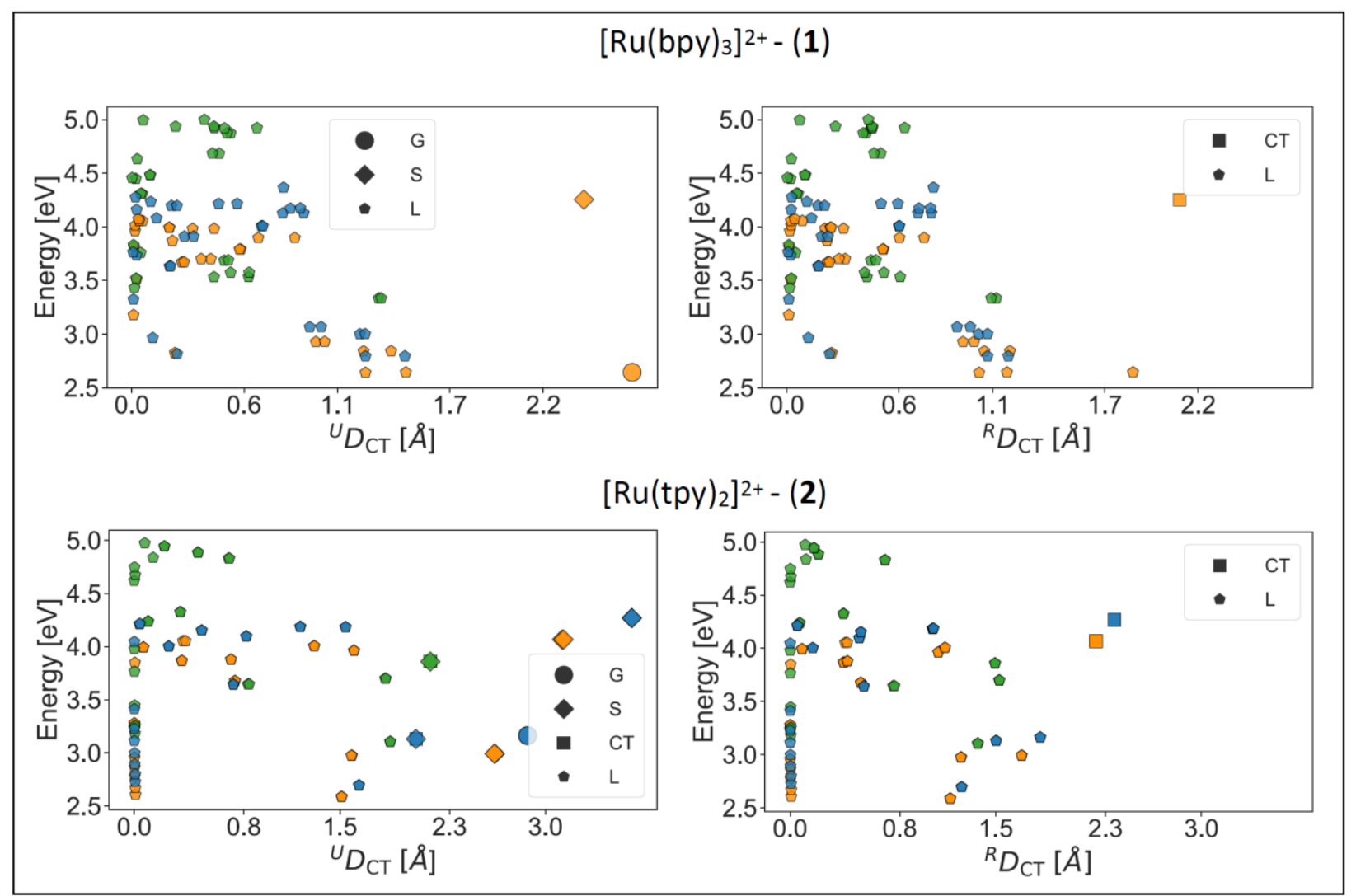

Figure 3: (left) ${ }^{\mathrm{U}} D_{\mathrm{CT}}$ and (right) ${ }^{\mathrm{R}} D_{\mathrm{CT}}$ values of the lowest thirty states of compounds $\mathbf{1}$ and $\mathbf{2}$, with respect to their energy computed with: B3LYP (orange), PBE0 (blue) and CAM-B3LYP (green). Each state is assigned a symbol, according to the $M_{\mathrm{AC}}$ diagnostic: dots for ghost states (G), diamonds for spurious states (S), squares for charge-transfer (CT) states, and pentagons for local excitations (L).

This becomes clear analyzing Figure 3 where diagnostic analysis based on both ${ }^{\mathrm{U}} M_{\mathrm{AC}}$ and ${ }^{\mathrm{R}} M_{\mathrm{AC}}$ of the lowest excitations of complexes $\mathbf{1}$ and $\mathbf{2}$ are depicted. Similar energy vs. $D_{\mathrm{CT}}$ plots for complexes 3, 4 and $\mathbf{5}$ can be found in Supporting information (Figure SI.13). Each excited state is assigned a label according to its $M_{\mathrm{AC}}$ value and represented using markers of different size and type. Transitions possessing ${ }^{\mathrm{U}} D_{\mathrm{CT}}$ values below $2.0 \AA$ are denoted as local (L), while those having $D_{\text {CT }}$ values $\mathrm{CT}$ greater or equal to $2.0 \AA$ are defined as charge-transfer (CT). Using this classification most MLCT transitions are actually classified as local since they involve density displacement on the ligand atoms closest to the metal center. Besides, transitions with excitation energy values lower than the corresponding $M_{\mathrm{AC}}$ are classified either as ghost (G) states, if their 
oscillator strength value is below 0.001 , or as spurious $(\mathrm{S})$ otherwise. Ghost states, which correspond to unphysical low-lying through-space transitions, are expected to appear mostly in the low energy/high $D_{\mathrm{CT}}$ range. For complexes $\mathbf{1}$ and $\mathbf{2}$ one would actually expect to find no ghost state even at GH level. Indeed, the CT character of their excited states is rather limited (with values of ${ }^{\mathrm{U}} D_{\text {Ст }}$ between $0.0 \AA$ and $3.1 \AA$ ).

As expected, only one state, namely the third one computed with the B3LYP functional- see Table SI. 1 is classified as $\mathrm{G}$ when using the ${ }^{\mathrm{U}} M_{\mathrm{AC}}$ index, but this same state is indeed detected as a local transition using the more refined description provided by the ${ }^{\mathrm{R}} M_{\mathrm{AC}}$ index. As a result, we can safely conclude that in the case of complex 1 all functionals considered provide a correct description of the excited states due to the limited range of charge transfer.

Analogously, also in the case of $\mathbf{2}$, only very few states are detected to be wrongly predicted when using the ${ }^{\mathrm{U}} M_{\mathrm{AC}}$ index two $\mathrm{G}$ at PBE0 level (S10 and S11), and few spurious states at B3LYP, PBE0 and CAM-B3LYP level - see Table SI.2. All these states, however, appear relatively high in energy, being all above $2.97 \mathrm{eV}$. Indeed, also in the case of complex $\mathbf{2}$, when using relaxed densities, no ghost or spurious states are detected in all excited states showing either a local or real CT character, as shown in the right panel of Figure 3. The difference between relaxed and unrelaxed density can be negligible or very important depending on the basis set and system considered as clearly shown by the comparison of left and right panels in Figure 3 suggesting that unrelaxed densities can be used for a first screening using the ${ }^{\mathrm{U}} M_{\mathrm{AC}}$ and that relaxed densities could then be used to analyze in further details the real nature of the so detected $\mathrm{G}$ and $\mathrm{S}$ states to get a definitive answer on their reliability.

The benefits of the $M_{\mathrm{AC}}$ diagnostic analysis are most appreciable when employed to investigate the electronic transitions of heteroleptic complexes, in which substituents of various kinds give rise to transitions of different character and CT extent. Complexes 3, 4 and $\mathbf{5}$ are exemplary in this regard as reported in Figure 4. Among the MLCTs, some involve the transfer of one electron from the metal center to the ancillary ligands (bipyridine, terpyridine). Others, most represented, involve the main ligand (dppz in $\mathbf{3}$, tpphz in $\mathbf{4}$ and pbbzim/tpy-HImzPh3 in 5). Further sub-classification of the MLCT transitions can be made considering the localization of the transferred charge on a specific part of the ligands - see for instance the list of structure and abbreviations reported in Figures SI.1 and SI.2 in Supporting Information. 
These variety of transitions extends also to ligand-to-ligand excitations (see for instance Figures SI.5 to SI.7 in Supporting Information which collect the ensemble of the NTOs associated to each vertical excitation computed with the different functionals). With the abundance of electronic transitions of different nature, the number of ghost and spurious states increases significantly.

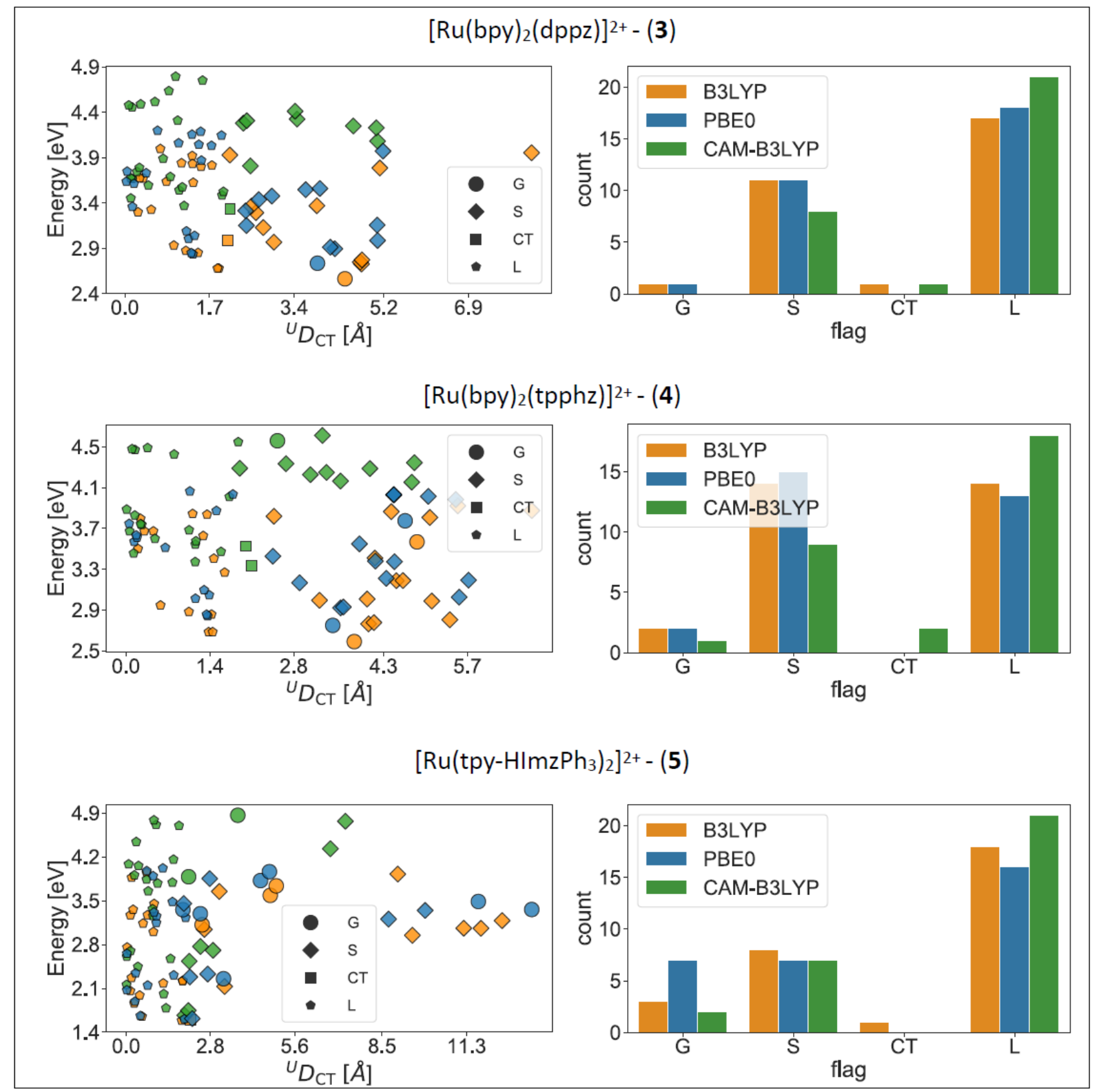

Figure 4: (left) ${ }^{\mathrm{U}} \mathrm{D}_{\mathrm{CT}}$ values of the lowest thirty states of compounds $\mathbf{3 , 4}$ and $\mathbf{5}$, with respect to their energy computed with: B3LYP (orange), PBE0 (blue) and CAM-B3LYP (green). Each state is assigned a symbol, according to the $M_{\mathrm{AC}}$ diagnostic: dots for ghost states $(\mathrm{G})$, diamonds for spurious states $(\mathrm{S})$, squares for charge-transfer (CT) states, and pentagons for local excitations (L). (right) Histograms displaying the number of excited states of each kind of transition at different levels of theory. 
As previously discussed for complexes $\mathbf{1}$ and $\mathbf{2}$, the use of ${ }^{\mathrm{U}} M_{\mathrm{AC}}$ allows a first screening of potentially non-physical states, whose real nature may be refined using relaxed densities and thus ${ }^{\mathrm{R}} M_{\mathrm{AC}}$. Analysis of the data collected in Tables SI. 3 to SI.5 and Figures SI.8 and SI.10 shows that for these complexes very few states differently classified using one or the other definition. In particular, among the 270 computed transitions across complexes $\mathbf{3 , 4}$ and $\mathbf{5}$, only 18 are characterized as S or $\mathrm{G}$ at ${ }^{\mathrm{U}} M_{\mathrm{AC}}$ level but not at ${ }^{\mathrm{R}} M_{\mathrm{AC}}$ one: only two states (ES16 and ES28) for complex $\mathbf{3}$ are misleadingly computed at ${ }^{\mathrm{U}} M_{\mathrm{AC}}$ level as Spurious (S) at B3LYP and one (ES14) at CAM-B3LYP and PBE0 level, and three states (ES18, ES21 and ES29) at CAM-B3LYP level for complex 4. In the case of complex 5 the number of states differently predicted is with the two approaches is slightly larger (1 ghost at the B3LYP level, six spurious states and one ghost at the CAM-B3LYP level and three spurious and one ghost at PBE0), but only three states appear at low energies, below $2.2 \mathrm{eV}$ - see Tables SI.3 to SI.5.

Due to these small differences in the following we will comment only on the descriptors computed using unrelaxed densities, since the ${ }^{\mathrm{U}} D_{\mathrm{CT}}$ index has the advantage of being computed on the fly without any extra cost.

The classification of the lowest thirty excited states reported in Figure 4 shows that for these systems the spurious and ghost states all fall into a particular range of $D_{\text {CT }}$ values, that is the ${ }^{\mathrm{U}} D_{\text {CT }}$ window between 2.2 and $7.5 \AA$ for complexes $\mathbf{3}$ and $\mathbf{4}$, and up to $12 \AA$ for $\mathbf{5}$, in accordance with the increased length of the molecule. Spurious and ghost states both appear in the same region of $D_{\text {Cт }}$ values, which prevents the identification of a clear limit of distinction of one or the other category in terms of CT extent only. Most of the states labeled as S or G in Figure 4 appear when applying GH functionals with low percentage of HF exchange, such as B3LYP and PBE0. The use of CAM-B3LYP drastically reduces their presence especially for complexes $\mathbf{3}$ and $\mathbf{4}$, as shown in the Figure 4 - right panel. Using GH, several states are identified as a ghost. We can appropriately comment on them inspecting their density change upon excitation. Consider, for instance, the first excited state (ES1) in complex $\mathbf{3}$, which, according to the $M_{\mathrm{AC}}$ analysis, is assigned as a ghost state when calculated using B3LYP and PBE0 (Table SI.3). The difference in density between ground and excited state of this last -reported in Figure SI.8 - shows that this state is an MLCT in which the excited electron is highly delocalized over the entire dppz ligand, while at CAM-B3LYP level the third excited state is computed to be more strongly localized on the tpy core of the dppz ligand. 
Thus, based on the $M_{\mathrm{AC}}$ analysis, we deduce that such an over-delocalization observed with GH is an artifact of the level of theory used, implying also that this state erroneously appears too low in energy. Analogous consideration applies to the first excited state (ES1) calculated using GH functionals for complex 4 (see Table SI.4 and Figure SI.9 in SI). On the other hand, in complex 5, the first ghost state appears higher in energy at both B3LYP and PBE0 level. At the latter level of theory, the first ghost is computed indeed at $550 \mathrm{~nm}(2.25 \mathrm{eV}$, see Table SI.5). The corresponding different density plots reported in Figure SI.10 show that at the GH level this is an MLCT transition, which extends over both pbbzim and tpy-HImzPh3 ligands. The same is true also for the ghost states computed at higher energy corresponding to $\mathrm{S} 17$ (at $3.29 \mathrm{eV}$ ) and $\mathrm{S} 21$ (at $3.35 \mathrm{eV}$ ) at PBE0 level. Overall, all these ghost states have the characteristic feature of being largely delocalized, with limited hole/particle overlap. Nonetheless ghost states do not contribute to alter the spectral shape. The case of the transitions classified as spurious is more controversial. These transitions correspond to excited states that are only predicted at -unphysically- lower energy but still present a non-zero oscillator strength thus contributing to the final shape of the absorption profiles. Correcting the energy positioning of these last shall thus influence the calculated spectra. Several excitations, mostly among the MLCT and ILCT/LLCT transitions of complexes $\mathbf{3}, \mathbf{4}$ and 5 are classified as spurious according to the $M_{\mathrm{AC}}$ diagnostics. A simple way of correcting the energy of these states is to consider the computed $M_{\mathrm{AC}}$ values as a lower bound estimate for the energy of both ghost and spurious states. Using this assumption, it is possible to recompute the absorption profiles of all complexes. Figure 5 shows the "corrected" profiles - calculated at the PBE0 level - where ghost and spurious states are shifted at -higher- energy, as determined by their corresponding $M_{\mathrm{AC}}$ value. The same analysis carried out at B3LYP and CAM-B3LYP level is reported in Supporting Information, Figures SI.11 and SI.12.

Independently on the functional, no spectral modification is expected for those complexes not displaying S states, i.e. in the case of $\mathbf{1}$ and $\mathbf{2}$, while larger effects appear for the absorption spectra of compounds $\mathbf{3}, \mathbf{4}$ and $\mathbf{5}$ calculated using GH, which have a larger number of spurious states.

Let's consider for instance complex 3: at PBE0 level as previously mentioned three transitions, corresponding to population of excited states 4, 9 and 10 and computed at $428 \mathrm{~nm}, 401 \mathrm{~nm}$ and $393 \mathrm{~nm}$ respectively, significantly contribute to the first absorption band, having oscillator strength values ranging between 0.15 and 0.23 . Based on the $M_{\mathrm{AC}}$ analysis (performed both using unrelaxed and relaxed densities, see Table S1.3) two of these excited states correspond to spurious states 
(ES4 and ES10). Both states correspond to MLCT transitions involving a large delocalization of the excited electron over the dppz ligand (see Figure SI.5 in Supporting information) and a sizable

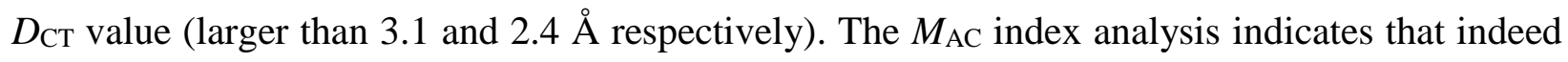
they should appear at higher energies: in the $218-278 \mathrm{~nm}$ range ES4 and in the $328-348 \mathrm{~nm}$ range ES10 based on the unrelaxed and relaxed corrections, respectively. Clearly both these $\mathrm{M}_{\mathrm{AC}}$ values are a lower bound to transition energies and as such they are intrinsically approximate and further errors are associated with the use of Eq. 2 to estimate them. Correcting the spectra on their basis determines the absence of an asymmetric tail in the first band, and a larger contribution to the second and third band in the spectra. 


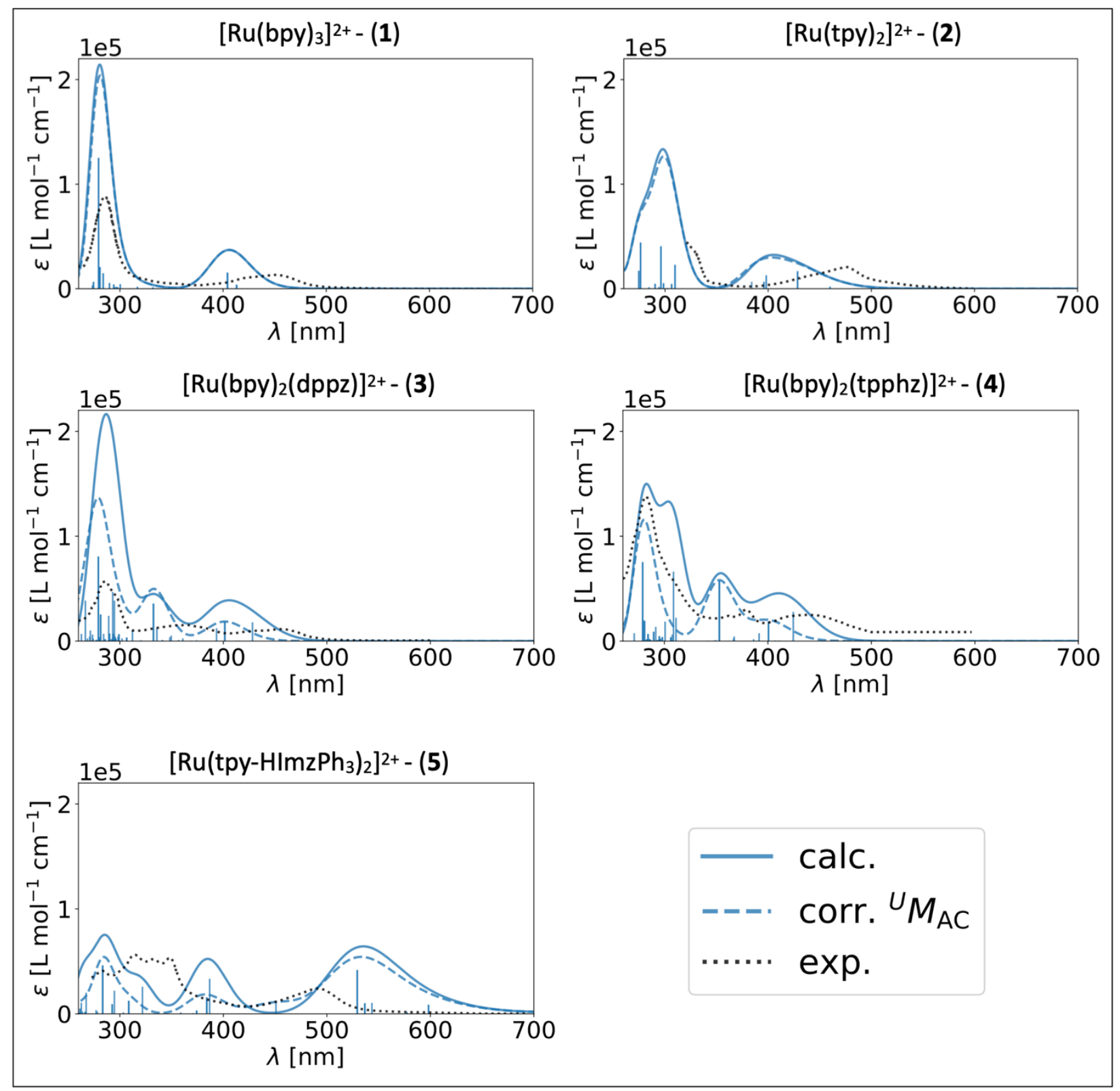

Figure 5: Absorption spectrum (solid lines) of 1, 2, 3, 4, and 5 computed at the PBE0 level of theory - see Supporting Information, along with the corresponding experimental spectra (dotted black line), retrieved from the literature. ${ }^{47,49-}$ ${ }^{52}$ The dotted lines correspond to corrected spectra where the spurious CT excitations are translated in the spectra at the wavelength corresponding to their $M_{\mathrm{AC}}$ index value.

The situation is exactly the same in the case of the spectrum predicted at B3LYP level (see Table SI.3 and Figure SI.5) two of the three electronic transition contributing to the first band involving also spurious states (ES4, predicted at $455 \mathrm{~nm}$, ES10 at $418 \mathrm{~nm}$ ). Again in this case these are two MLCT transitions showing a large delocalization over the dppz ligand and high $D_{\mathrm{CT}}$ values that, based on the $\mathrm{M}_{\mathrm{AC}}$ indexes, should appear at higher energies (in the 206-257 $\mathrm{nm}$ and in the 452- 
$436 \mathrm{~nm}$ range, respectively). On the hand, at CAM-B3LYP level no spurious transitions are found among the ones contributing to the spectra in the UV-Vis range, thus no correction is applied. Of note, by construction corrections, if present, always determine a blueshift of the transition energies. The situation for complex $\mathbf{4}$, is qualitatively similar: of the three transitions contributing to the first band computed at PBE0 level (at $417 \mathrm{~nm}, 401 \mathrm{~nm}$ and $353 \mathrm{~nm}$ ) only the first one corresponds to a spurious state (see Table SI.4) and should appear in the 241-300 nm range (see Table SI.4). One again the transition corresponds to a state (ES4) characterized by a high $D_{\mathrm{CT}}$ and a significant delocalization on the tppz ligand (see Figure SI.6). A similar analysis and conclusion can be drawn for B3LYP computed transitions while using CAM-B3LYP the most intense transition at $357 \mathrm{~nm}$, which gives rise to the first absorption band is not a spurious state and, while also possessing an MLCT character (see Figure SI.6) it is actually involving only the tetraazatriphenylene moiety (Tat see scheme 2 in Supporting Information) of the tppz ligand and is thus showing a smaller CT character $\left(D_{\mathrm{CT}}\right.$ of $1.5 \AA$, see Table SI.4).

Interestingly, while one could expect that due to their lower energies, the lowest transitions of 5 could be related to spurious states, this is actually not the case. Indeed, as shown also by the right column of Figure 4, the number of spurious states of $\mathbf{5}$, both at GH and RSH level, is very limited compared to compounds $\mathbf{3}$ and $\mathbf{4}$, despite the larger size of complex 5. Indeed, many of the computed transitions, while possessing an MLCT character (see Figure SI.7) are actually characterized by relatively low CT distances (see data in Table SI.5) and are therefore defined as Local (L) in Figure 4.

As a consequence, the most intense transition contributing to the first absorption band at both B3LYP and PBE0 (that is the one associated with ES11) is not involving a spurious state as it corresponds to an MCLT transition with a very limited CT character and localized on the tpy ligand. The same holds for CAM-B3LYP. Therefore, for this system the first band is not affected by the correction unlike the second peak in the spectrum (predicted around $400 \mathrm{~nm}$ ), which results from several closely lying spurious states, presenting larger $\mathrm{CT}$ values and involving more delocalized orbitals.

\section{Conclusions.}

We have shown how, in the case of simple TMC, the presence of ghost and spurious states cannot be excluded using hybrid functionals and may affect the interpretation of both photophysical and 
photochemical processes. A diagnostic analysis tool, the $M_{\mathrm{AC}}$ index, that can be used for their detection and correction has been proposed and proven effective to assess the quality of the excited states also in the case of TMCs. The analysis performed shows that spurious and ghost states can appear in a wide spectral range and that it is difficult to detect them only on the basis of the CT length or of the degree of delocalization of the state. Indeed, neither very delocalized nor charge transfer states are necessarily spurious or ghost, this criteria being alone neither a necessary nor a sufficient condition to identify a state as spurious. An index, as the $M_{\mathrm{AC}}$ index, combining both information on the degree of locality and CT, can thus provide relevant insights to detect unphysical CT states.

Of note, the evaluation of the $M_{\mathrm{AC}}$ index is rather computationally inexpensive. We based our discussion on unrelaxed densities, since the classification of the excited-state levels can be made on the fly and for all transitions at once in such a case. Relaxed densities provide nonetheless a refined description of excited states possessing a sizable CT character. ${ }^{68}$ Using a suitably large basis set reduces the difference between the description provided using relaxed and unrelaxed densities. ${ }^{68}$ Nonetheless since relaxation effects are expected to be also dependent on the system considered a careful case-by-case examination based on relaxed density of all the spurious and ghost states predicted at unrelaxed density level is strongly encouraged especially when aiming at using the $M_{\mathrm{AC}}$ values to correct the computed spectra.

Supporting Information. Tables reporting all raw rata computed with the different strategies for all compounds, including excitation energies, oscillator strength $f_{\mathrm{osc}},{ }^{\mathrm{U}} D_{\mathrm{CT}}$ and ${ }^{\mathrm{R}} D_{\mathrm{CT}},{ }^{\mathrm{U}} M_{\mathrm{AC}}$ and ${ }^{\mathrm{R}} M_{\mathrm{AC}}$, and relative labels of the lowest thirty excited states. A selection of natural transition orbitals and difference density plots. Absorption spectra and ${ }^{\mathrm{R}} D_{\mathrm{CT}}$ vs. energy, as referenced in the main text.

\section{Acknowledgement}

I.C, F.M., M.C., and J.S.G gratefully acknowledge the European Research Council (ERC) for funding (ERC Consolidator Grant STRIGES to I.C., GA No 648558). M. Casida is acknowledged for interesting discussions during the CECAM workshop Theoretical and Computational Inorganic Photochemistry: Methodological Developments, Applications and Interplay with Experiments Toulouse (France).

Data Availability Statement : The data that supports the findings of this study are available within the article and its supplementary material. 


\section{References}

1. Cramer, C. J.; Truhlar, D. G. Density Functional Theory for Transition Metals and Transition Metal Chemistry. Phys. Chem. Chem. Phys. 2009, 11, 10757-10816.

2. Daniel, C. Photochemistry and Photophysics of Transition Metal Complexes: Quantum Chemistry. Coord. Chem. Rev. 2015, 282-283, 19-32.

3. Daniel, C. Absorption Spectroscopy, Emissive Properties, and Ultrafast Intersystem Crossing Processes in Transition Metal Complexes: TD-DFT and Spin-Orbit Coupling. Top. Curr. Chem. 2016, $368,377-413$.

4. Francés-Monerris, A.; Gros, P. C.; Assfeld, X.; Monari, A.; Pastore, M. Toward Luminescent Iron Complexes: Unravelling the Photophysics by Computing Potential Energy Surfaces. ChemPhotoChem 2019, 3 (9), 666-683.

5. Cordones, A.A.; Lee, J. H. ; Hong, K.; Cho, H.; Garg, K. ; Boggio-Pasqua, M.; Rack, J. J. ; Huse, N. ; Schoenlein, R. W. ; Kim T. K. Transient metal-centered states mediate isomerization of a photochromic ruthenium-sulfoxide complex Nat Commun 2018, 9, 1989.

6. Talotta, F. Heully, J.L. Alary, F., Dixon, I. M., Gonzalez, L. Boggio-Pasqua, M. Linkage Photoisomerization Mechanism in a Photochromic Ruthenium Nitrosyl Complex: New Insights from an MS-CASPT2 Study J. Chem. Theory Comput. 2017, 13, 6120-6130

7. Vlček, A.; Zálišs, S. Modeling of Charge-Transfer Transitions and Excited States in d6 Transition Metal Complexes by DFT Techniques. Coord. Chem. Reviews 2007, 251, 258-287.

8. Raucci, U.; Ciofini, I.; Adamo, C.; Rega N. Unveiling the Reactivity of a Synthetic Mimic of the Oxygen Evolving Complex J Chem Phys Lett 2016, 7, 5015-5021

9. Mai, S.; Plasser, F.; Dorn, J.; Fumanal, M.; Daniel, C.; González, L. Quantitative Wave Function Analysis for Excited States of Transition Metal Complexes. Coord. Chem. Rev. 2018, 361, 74-97.

10. Roundhill, D. M. Photochemistry and Photophysics of Metal Complexes; Springer, Boston, MA, 1994.

11. Juris, A.; Balzani, V.; Barigelletti, F.; Campagna, S.; Belser, P.; von Zelewsky, A. Ru(II) Polypyridine Complexes: Photophysics, Photochemistry, Eletrochemistry, and Chemiluminescence. Coord. Chem. Rev. 1988, 84, 85-277.

12. Campagna, S.; Puntoriero, F.; Nastasi, F.; Bergamini, G.; Balzani, V. Photochemistry and Photophysics of Coordination Compounds: Ruthenium. In Photochemistry and Photophysics of Coordination Compounds I; Balzani, V., Campagna, S., Eds.; Springer Berlin Heidelberg: Berlin, Heidelberg, 2007; pp 117-214.

13. Grätzel, M. Dye-Sensitized Solar Cells. J. Photochem. Photobiol. C: Photochem. Rev. 2003, 4 (2), $145-153$.

14. Hagfeldt, A.; Boschloo, G.; Sun, L.; Kloo, L.; Pettersson, H. Dye-Sensitized Solar Cells. Chem. Rev. 2010, 110 (11), 6595-6663.

15. Su, J.; Zhu, T.; Pauporté, T.; Ciofini, I.; Labat, F. Improving the Heterointerface in Hybrid OrganicInorganic Perovskite Solar Cells by Surface Engineering: Insights from Periodic Hybrid Density Functional Theory Calculations J Comput Chem 2020, 41, 1740-1747.

16. Labat, F.; Le Bahers, T.; Ciofini, I.; Adamo C. First-principles modeling of dye-sensitized solar cells: challenges and perspectives Acc. Chem. Research 2012, 45, 1268-1277.

17. Labat, F.; Ciofini, I.; Hratchian, H.; Frisch, M.; Raghavachari, K.; Adamo C. Insights into working principles of $\mathrm{N} 3 / \mathrm{TiO}_{2}$ dye-sensitized solar cells from first principles modeling J. Phys. Chem. C 2011, $115,4297-4306$

18. Knoll, J. D.; Turro, C. Control and Utilization of Ruthenium and Rhodium Metal Complex Excited States for Photoactivated Cancer Therapy. Coord. Chem. Rev. 2015, 282-283, 110-126.

19. Ruggiero, E.; Castro, S. A.; Habtemariam, A.; Salassa, L. The Photochemistry of Transition Metal Complexes and Its Application in Biology and Medicine. Structure and Bonding 2014, 165, 69-107.

20. Poynton, F. E.; Bright, S. A.; Blasco, S.; Williams, D. C.; Kelly, J. M.; Gunnlaugsson, T. The Development of Ruthenium(ii) Polypyridyl Complexes and Conjugates for in Vitro Cellular and in Vivo Applications. Chem. Soc. Rev. 2017, 46 (24), 7706-7756. 
21. Monro, S.; Colón, K. L.; Yin, H.; Roque, J., 3rd; Konda, P.; Gujar, S.; Thummel, R. P.; Lilge, L.; Cameron, C. G.; McFarland, S. A. Transition Metal Complexes and Photodynamic Therapy from a Tumor-Centered Approach: Challenges, Opportunities, and Highlights from the Development of TLD1433. Chem. Rev. 2019, 119 (2), 797-828.

22. Karges, J.; Heinemann, F..; Jakubaszek, M; Maschietto, F.; Subecz, C.; Dotou, M.; Blacque, O.; Tharaud, M.; Goud, B.; Viñuelas Zahínos, E.; Spingler, B.; Ciofini, I. Gasser, G. Rationally Designed Long-Wavelength Absorbing Ru(II) Polypyridyl Complexes as Photosensitizers for Photodynamic Therapy J Am. Chem. Soc. 2020, 142, 6578-6587

23. Karges, J.; Kuang, S.; Maschietto, F.; Blacque, O. Ciofini, I.. Chao H.; Gasser G. Ruthenium Complexes for 1- and 2-Photon Photodynamic Therapy: From In Silico Prediction to In Vivo Applications Nature Comm 2020, 11, 3262.

24. Roos, B. O.; Taylor, P. R.; Sigbahn, P. E. M. A Complete Active Space SCF Method (CASSCF) Using a Density Matrix Formulated Super-CI Approach. Chem. Phys. 1980, 48, 157-173.

25. Roos, B. O.; Lindh, R.; Malmqvist, P. Å; Veryazov, V.; Widmark, P.-O. Multiconfigurational Quantum Chemistry; John Wiley \& Sons, 2016.

26. Andersson, K.; Malmqvist, P.; Roos, B. O. Second-order Perturbation Theory with a Complete Active Space Self-consistent Field Reference Function. J. Chem. Phys. 1992, 96, 1218-1226.

27. Fumanal, M.; Daniel, C. Description of Excited States in [Re(Imidazole)(CO)3 (Phen)](+) Including Solvent and Spin-Orbit Coupling Effects: Density Functional Theory versus Multiconfigurational Wavefunction Approach. J. Comput. Chem. 2016, 37 (27), 2454-2466.

28. Furche, F.; Rappoport, D. Density Functional Methods for Excited States: Equilibrium Structure and Electronic Spectra. Theoretical and Computational Chemistry. 2005, 16, 93-128.

29. Adamo, C.; Jacquemin, D. The calculations of excited-state properties with Time-Dependent Density Functional Theory Chem. Soc. Rev. 2013, 42, 845-856

30. Dreuw, A.; Head-Gordon, M. Failure of Time-Dependent Density Functional Theory for Long-Range Charge-Transfer Excited States: The Zincbacteriochlorin-Bacteriochlorin and BacteriochlorophyllSpheroidene Complexes. J. Am. Chem. Soc. 2004, 126 (12), 4007-4016.

31. Dreuw, A. ; Weisman, J. L. ; Head-Gordon, M. Long-range charge-transfer excited states in timedependent density functional theory require non-local exchange J. Chem. Phys. 2003, 119, 2943.

32. Ciofini, I.; Chermette, H.; Adamo, C. A Mean-Field Self-Interaction Correction in Density Functional Theory: Implementation and Validation for Molecules. Chem. Phys. Lett. 2003, 380, 12-20.

33. Ciofini, I.; Adamo, C.; Chermette, H. Self-Interaction Error in Density Functional Theory: A MeanField Correction for Molecules and Large Systems. Chem. Phys. 2005, 309 67-76. https://doi.org/10.1016/j.chemphys.2004.05.034.

34. Casida, M. E.; Jamorski, C.; Casida, K. C.; Salahub, D. R. Molecular Excitation Energies to HighLying Bound States from Time-Dependent Density-Functional Response Theory: Characterization and Correction of the Time-Dependent Local Density Approximation Ionization Threshold. J. Chem. Phys. 1998, 108, 4439-4449.

35. Grimme, S.; Parac, M. Substantial Errors from Time-Dependent Density Functional Theory for the Calculation of Excited States of Large $\pi$ Systems. Chem.Phys.Chem. 2003, 4, 292-295.

36. Tozer, D. J. Relationship between Long-Range Charge-Transfer Excitation Energy Error and Integer Discontinuity in Kohn-Sham Theory. J. Chem. Phys. 2003, 119, 12697-12699.

37. Mardirossian, N.; Head-Gordon, M. Thirty Years of Density Functional Theory in Computational Chemistry: An Overview and Extensive Assessment of 200 Density Functionals. Mol. Phys. 2017, 115 (19), 2315-2372.

38. Ottochian, A.; Morgillo, C.; Ciofini, I.; Frisch, M. J.; Scalmani, G.; Adamo, C. Double Hybrids and Time-dependent Density Functional Theory: An Implementation and Benchmark on Charge Transfer Excited States. J. Comput. Chem. 2020, 41, 1242-1251.

39. Casanova-Páez, M.; Goerigk, L. Assessing the Tamm-Dancoff Approximation, Singlet-singlet, and Singlet-triplet Excitations with the Latest Long-Range Corrected Double-Hybrid Density Functionals. J. Chem. Phys. 2020, 153 (6), 064106. 
40. Baer, R.; Livshits, E.; Salzner, U. Tuned Range-Separated Hybrids in Density Functional Theory. Annu. Rev. Phys. Chem. 2010, 61, 85-109.

41. Manna, A. K.; Lee, M. H.; McMahon, K. L.; Dunietz, B. D. Calculating High Energy Charge Transfer States Using Optimally Tuned Range-Separated Hybrid Functionals. J Chem. Theory Comput. 2015, $11,1110-1117$.

42. Cai, Z.-L.; Sendt, K.; Reimers, J. R. Failure of Density-Functional Theory and Time-Dependent Density-Functional Theory for Large Extended $\pi$ Systems. J. Chem. Phys. 2002, 117 5543-5549

43. Head-Gordon, M., Grana, A. M., Maurice, D., White, C. A. Analysis of electronic transitions as the difference of electron attachment and detachment densities. J. Phys. Chem. 1995, 99 (39), 1426114270.

44. Peach, M. J. G. ; Benfield, P. ; Helgaker, T. ; Tozer, D. J. Chem. Phys. 2008, 128, 044118.

45. Bahers, T. L.; Le Bahers, T.; Adamo, C.; Ciofini, I. A Qualitative Index of Spatial Extent in ChargeTransfer Excitations. J Chem. Theory Comput. 2011, 7, 2498-2506.

46. Guido, C. A.. ; Cortona, P. ; Mennucci, B. ; Adamo, C. J. Chem. Theory Comput. 2013, 9, 3118.

47. Plasser, F.;Wormit, M.; Dreuw, A. New tools for the systematic analysis and visualization of electronic excitations. I. Formalism. J. Chem. Phys. 2014, 141, 024106

48. Etienne, T. ; Assfeld X. ; Monari A Toward a Quantitative Assessment of Electronic Transitions' Charge-Transfer Character, J. Chem. Theory Comput. 2014, 10, 3896-3905

49. Adamo, C. ; Le Bahers, T. ; Savarese, M. ; Wilbraham, L. ; Garcia, G. ; Fukuda, R. ; Ehara, M. ; Rega, N. ; Ciofini, I. Coord. Chem. Rev. 2015, 304, 166.

50. Plasser, F. TheoDORE: A toolbox for a detailed and automated analysis of electronic excited state computations J. Chem. Phys. 152, 084108 (2020)

51. Huet, L.; Perfetto, A.; Muniz-Miranda, F.; Campetella, M.; Adamo, C.; Ciofini, I. A general densitybased index to analyze charge transfer phenomena: from models to butterfly molecules $\mathrm{J}$ Chem Theory Comput 2020, 16, 4543-4553

52. Martin, R. L. Natural Transition Orbitals. J. Chem. Phys. 2003, 118, 4775-4777.

53. Coe, B.J., Avramopoulos, Papadopoulos, M.G., Pierloot, K., Vancoillie, S., Reis. H., Theoretical Modelling of Photoswitching of Hyperpolarisabilities in Ruthenium Complexes. Chem. Eur. J. 2013, 19 (47), 15955-15963.

54. De Sousa, S., Ducasse, L., Kauffmann, B., Toupance, T., Olivier, C., Functionalization of a Ruthenium-Diacetylide Organometallic Complex as a Next-Generation Push-Pull Chromophore. Chem. Eur. J, 2014, 20 (23), 7017-7024.

55. De Sousa, S., Lyu, S., Ducasse, L, Toupance, T., Olivier, C., Tuning visible-light absorption properties of Ru-diacetylide complexes: simple access to colorful efficient dyes for DSSCs. J. Mater. Chem. A 2015, 3 (35), 18256-18264.

56. Olaru, M., Rychagova, E., Ketkov, S., Shynkarenko, Y., Yakunin, S., Kovalenko, M.V., Yablonskiy, A., Andreev, B., Kleemiss, F., Beckmann, J., Vogt. M., A Small Cationic Organo-Copper Cluster as Thermally Robust Highly Photo- and Electroluminescent Material. J. Am. Chem. Soc. 2020, 142 (1), 373-381.

57. Le Bahers, T.; Brémond, E.; Ciofini, I.; Adamo A. Nature of vertical excited states of dyes containing metals for DSSCs applications: insights from TD-DFT and density based indexes Phys Chem Chem Phys 2014, 16, 14435-14444

58. Yoshimura, A.; Hoffman, M. Z.; Sun, H. An Evaluation of the Excited State Absorption Spectrum of $\mathrm{Ru}(\mathrm{bpy}) 32+$ in Aqueous and Acetonitrile Solutions. J. Photochem. Photobiol. A Chem. 1993, 70 (1), 29-33.

59. Jakubikova, E.; Chen, W.; Dattelbaum, D. M.; Rein, F. N.; Rocha, R. C.; Martin, R. L.; Batista, E. R. Electronic Structure and Spectroscopy of $[\mathrm{Ru}(\mathrm{tpy}) 2] 2, \quad[\mathrm{Ru}(\mathrm{tpy})(\mathrm{bpy})(\mathrm{H} 2 \mathrm{O})] 2$, and $[\mathrm{Ru}($ tpy)(bpy)(Cl)]. Inorg. Chem. 2009, 48, 10720-10725.

60. McKinley, A. W.; Lincoln, P.; Tuite, E. M. Environmental Effects on the Photophysics of Transition Metal Complexes with Dipyrido [2, 3-a: 3', 2'-C] Phenazine (dppz) and Related Ligands. Coord. Chem. Rev. 2011, 255, 2676-2692. 
61. Kobayashi, K.; Ohtsu, H.; Nozaki, K.; Kitagawa, S.; Tanaka, K. Photochemical Properties and Reactivity of a Ru Compound Containing an NAD/NADH-Functionalized 1,10-Phenanthroline Ligand. Inorg. Chem. 2016, 55 (5), 2076-2084.

62. Torieda, H.; Nozaki, K.; Yoshimura, A.; Ohno, T. Low Quantum Yields of Relaxed Electron Transfer Products of Moderately Coupled Ruthenium(II)-Cobalt(III) Compounds on the Subpicosecond Laser Excitation. J. Phys Chem. A. 2004, 108, 4819-4829.

63. Sun, Y.; Collins, S. N.; Joyce, L. E.; Turro, C. Unusual Photophysical Properties of a ruthenium(II) Complex Related to [Ru(bpy)2(dppz)]2+. Inorg. Chem. 2010, 49 (9), 4257-4262.

64. Bhaumik, C.; Das, S.; Maity, D.; Baitalik, S. Luminescent Bis-Tridentate Ruthenium(ii) and Osmium(ii) Complexes Based on Terpyridyl-Imidazole Ligand: Synthesis, Structural Characterization, Photophysical, Electrochemical, and Solvent Dependence Studies. Dalton Trans. 2012, 41, 2427-2438.

65. Campetella, M.; Maschietto, F.; Frisch, M. J.; Scalmani, G.; Ciofini, I.; Adamo, C. Charge Transfer Excitations in TDDFT: A Ghost-Hunter Index. J Comput. Chem. 2017, 38, 2151-2156.

66. Mulliken, R. S. Molecular Compounds and Their Spectra. II. J. Am. Chem. Soc. 1952, 74, 811-824.

67. Casida, M. E. Time-Dependent Density Functional Response Theory for Molecules. Recent Advances in Density Functional Methods. 1995, pp 155-192.

68. Maschietto, F.; Campetella, M.; Frisch, M. J.; Scalmani, G.; Adamo, C.; Ciofini, I. How Are the Charge Transfer Descriptors Affected by the Quality of the Underpinning Electronic Density? $J$ Comput. Chem. 2018, 39, 735-742.

69. Pastore, M.; Assfeld, X.; Mosconi, E.; Monari, A.; Etienne, T. Unveiling the Nature of Post-Linear Response Z-Vector Method for Time-Dependent Density Functional Theory. J. Chem. Phys. 2017, 47024108.

70. Handy, N. C.; Schaefer, H. F. On the Evaluation of Analytic Energy Derivatives for Correlated Wave Functions. J. Chem. Phys. 1984, 81, 5031-5033.

71. Furche, F.; Ahlrichs, R. Adiabatic Time-Dependent Density Functional Methods for Excited State Properties. J. Chem. Phys. 2002, 117, 7433-7447.

72. Frisch, M. J. et al. Gaussian 16 Revision B.01;2016.

73. Cossi, M.; Barone, V. Time-dependent density functional theory for molecules in liquid solutions. $J$. Chem. Phys 2001, 115, 4708-4717.

74. Zhao, H. C.; Fu, B.-L.; Schweinfurth, D.; Harney, J. P.; Sarkar, B.; Tsai, M.-K.; Rochford, J. Tuning Oxyquinolate Non-Innocence at the Ruthenium Polypyridyl Core. Eur. J. Inorg. Chem. 2013, 25, 4410-4420

75. Becke, A. D. Density-functional thermochemistry. III. The role of exact exchange. J. Chem. Phys 1993, 98, 5648-5652.

76. Lee, C.; Yang, W.; Parr, R. G. Development of the Colle-Salvetti correlation-energy formula into a functional of the electron density. Phys. Rev. B 1988, 37, 785-789.

77. Hariharan, P. C.; Pople, J. A. The influence of polarization functions on molecular orbital hydrogenation energies. Theo. Chim.Acta 1973, 28, 213-222.

78. Roy, L. E.; Hay, P. J.; Martin, R. L. Revised Basis Sets for the LANL Effective Core Potentials. $J$ Chem. Theory Comput. 2008, 4, 1029-1031.

79. Yanai, T.; Tew, D. P.; Handy, N. C. A new hybrid exchange correlation functional using the Coulomb-attenuating method (CAM-B3LYP). Chem. Phys. Lett. 2004, 393, 51-57.

80. Adamo, C.; Barone, V. Toward reliable density functional methods without adjustable parameters: The PBE0 model. J. Chem. Phys. 1999, 110, 6158-6170.

81. Chantzis, A., Very, T., Monari, A., Assfeld, X. Improved Treatment of Surrounding Effects: UV/vis Absorption Properties of a Solvated Ru(II) Complex. J. Chem. Theory Comput. 2012 8, 1536-1541. 